\title{
Research Paper: \\ Assessing the Validity and Reliability of the Persian Version of the Interper- sonal Problem Solving Skills Assessment Tool in Schizophrenia
}

\author{
Milad Abolhasani ${ }^{1}$ (D) ${ }^{*}$ Ashraf Karbalaee Nouri ${ }^{1}$ (D), Enayatollah Bakhshi ${ }^{2}$ (D) Mojtaba Kheirkhah ${ }^{1}$ (D) \\ 1. Department of Occupational Therapy, University of Social Welfare and Rehabilitation Sciences, Tehran, Iran. \\ 2. Department of Biostatistics, University of Social Welfare and Rehabilitation Sciences, Tehran, Iran
}

\begin{tabular}{|c|c|}
\hline $\begin{array}{l}\text { Use your device to scan } \\
\text { and read the article online }\end{array}$ & \\
\hline afiping & $\begin{array}{l}\text { Version of the Interpersonal Problem Solving Skills Assessment Tool in Schizophrenia (Persian)]. Archives of Rehabilitation. } \\
\text { 2021; 22(1):118-131. https://doi.org/10.32598/RJ.22.1.3214.1 }\end{array}$ \\
\hline 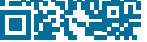 & Ol'https://doi.org/10.32598/RJ.22.1.3214.1 \\
\hline
\end{tabular}

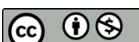

Received: 15 Jun 2020 Accepted: 29 Oct 2020 Available Online: 01 Apr 202

Keywords:

Schizophrenia, Social skills, Validity, Reliability, Translation

\begin{abstract}
A B STRACT
Objective This study aimed to translate the Assessment of Interpersonal Problem-Solving Skills (AIPSS) into Persian and to evaluate the validity and reliability of the Persian version of AIPSS to use for adults with schizophrenia.

Materials \& Methods In this methodological study, the translation process was performed according to the International Quality of Life Assessment (IQOLA) protocol. The face validity of the translated AIPSS was determined based on the opinions of experts and The Content Validity Index (CVI) and Content Validity Ratio (CVR) were also calculated for each item. The Persian version of the test was performed on 52 patients with schizophrenia disorders at Tehran's Razi Mental Hospital; they were selected using a convenience sampling method. Cronbach's alpha coefficient was used to evaluate internal consistency. Inter-rater reliability was determined by the Intraclass Correlation Coefficient (ICC). A retest was complete on 15 patients with 2 weeks interval and ICC was used to determine the test-retest reliability. Results Face validity was confirmed by the experts' opinions. The Content Validity Ratio (CVR) and the Content Validity Index (CVI) were equal to one for all scenes. Cronbach's alpha coefficient for all scales was ranged between 0.511 and 0.821 . The ICC in all scales were more than 0.98 for inter-rater reliability. In calculating test-retest reliability, the ICC for all scales ranged 0.733-0.893.

Conclusion Results show that the Persian version of AIPSS has acceptable face validity, content validity, internal consistency, inter-rater reliability test-retest reliability. Therefore, this instrument can be used in clinical fields and research studies to assess the social skills of Iranian patients with schizophrenia.
\end{abstract}

\section{Extended Abstract}

\section{Introduction}

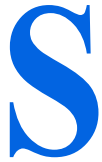

chizophrenia is a severe mental disorder and one of the leading causes of disability in the world [1]. The annual prevalence of schizophrenia varies but averages 15 per 100,000 [2]. Symptoms of schizophrenia include delirium, hallucinations, thought disorders, speech disorders, behavioral disorders, indifference, and sluggish emotional responses [3].
Defects in social functioning and social skills are often associated with the diagnosis of schizophrenia [4-7]. In a study conducted in Iran on 208 schizophrenia patients, $95.75 \%$ of them had social dysfunction $[2,3]$. Other studies have reported social dysfunction in 50 and $87 \%$ of patients with schizophrenia [10].

Social function is a general term that refers to an individual's ability to interact appropriately and effectively in society [11]. The ability to solve an interpersonal problem is one of the main aspects of social skills that many patients with schizophrenia suffer from $[12,13]$.

\section{* Corresponding Author:}

Ashraf Karbalaee Nouri, PhD.

Address: Department of Occupational Therapy, University of Social Welfare and Rehabilitation Sciences, Tehran, Iran.

Tel: +98 (21) 22180037

E-Mail: nouri.uswr@gmail.com 
Social dysfunction is one of the debilitating and resistant aspects of schizophrenia. Social problems in adulthood can lead to severe disruption in functional role areas (such as marriage, employment, and friendship) [14]. Impairment in social functioning also affects the quality of life [15] and predicts outcomes, such as relapse, poor course of illness, and unemployment [16-18].

The emphasis on improving social skills as a therapeutic goal for schizophrenia creates the need for an appropriate assessment tool with good psychometric conditions. It is necessary to have an assessment tool to determine the basic level of the individual's skills, strengths and weaknesses, and the patient's progress so that the therapist can provide a suitable rehabilitation program for the patient.

The Assessment of Interpersonal Problem-Solving Skills (AIPSS) tool is a role-playing tool that assesses the social skills of patients with schizophrenia based on interpersonal problem-solving $[19,20]$. This tool was introduced by Donahue et al. in 1990 and has been translated into French [21], Turkish [22], Chinese [23], Swedish [24], and Norwegian [25] and has been used in various studies [21, 26-30]. This tool appears to be a sensitive tool and reveals problems in schizophrenia that other tools cannot show [26]. AIPSS differs from other common tools in assessing social performance for the following reasons: 1 . Using observational role-playing instead of self-declaration or third-party reporting; 2- Determining the current rank of the patient instead of his past performance; 3- using real-life video scenes related to the patient's ability to solve social problems; and 4- Assessing the patient's social perception and processing social information to plan verbal and non-verbal social actions and responses [27]. In this study, the validity and reliability of the Persian version of this tool were examined.

\section{Materials and Methods}

This research was a methodological study and the statistical population included Iranian (adult) patients with schizophrenia referring to the Razi Psychiatric Hospital in Tehran in 2019. A total of 52 individuals were selected by available sampling according to the inclusion and exclusion criteria to participate in the study.

Inclusion criteria were as follows: 1- Schizophrenia according to the fifth version of the Guidelines for Diagnosis and Statistics of Mental Disorders, which is listed in the medical record by a psychiatrist; 2- the Age of over 18 years old; 3- Having full consent to participate in the study; and 4- the lack of other disorders in their medical records. The exclusion criteria were: 1 - severe visual or hearing impair- ment 2- severe impairment in reality testing 3- Recurrence of symptoms during the performance, and 4- Unwillingness to continue cooperation.

\section{Procedure}

Persian version preparation process

A Persian version was prepared based on the International Bureau of Evaluation of Quality of Life Assessment. To reach the Persian version of the evaluation scenes, first, in a meeting with the presence of researchers, the Persian copy of the evaluation scenes obtained in the previous stage was examined for cultural adaptation and using this copy, the evaluation scenes were filmed. The film was then reviewed by 7 psychologists. To compare the two versions, a form was provided that included the following factors: environment, speech quality, non-verbal components, and verbal content. For each of these cases, the question was asked whether there was a major discrepancy between the original and the reconstructed scene. Also, for each scene, to check the cultural compatibility, a question entitled "Is the Persian version culturally applicable for the Iranian society" was included. Experts' suggestions were used to improve the evaluation scenes.

\section{Examining the apparent and content validity}

The apparent validity of the test evaluation scenes was assessed using a survey of 7 experienced and knowledgeable experts in psychology, who examined the evaluation scenes in terms of clarity and simplicity. To determine the content validity, the Lavash method was used to calculate the Content Validity Index (CVI) and Content Validity Ratio (CVR). For this purpose, the experts rated the content of the test items in terms of relevance and necessity, and finally, the preliminary test was performed on 3 healthy people and 2 patients to clarify the ambiguous parts.

\section{Tools}

Interpersonal information questionnaire: Demographic information included education level, gender, age, marital status, and length of hospital stay in the participating hospital. This information was obtained through the subjects' reports or their medical records.

AIPSS: This tool was designed to assess social skills in people with schizophrenia using the 1990 problem-solving model by Donahue et al. AIPSS is done using a film that consists of 14 short scenes of interaction between two actors. The scenes are used to familiarize the examiner with the testing process. There are no problems in three of these 
Table 1. Cronbach's alpha results and intra-rank correlation coefficient

\begin{tabular}{ccccc}
\hline \multirow{2}{*}{ Scale } & Cronbach's Alpha & \multicolumn{2}{c}{ Intra-door Correlation Coefficient } & \multirow{2}{*}{ Number of Items } \\
\cline { 3 - 5 } & & Between Two Evaluators (Number = 22) & Open Test (Number =15) & \\
\hline ID & 0.511 & 0.996 & 0.733 & 13 \\
\hline Dissection & 0.675 & 0.982 & 0.893 & 10 \\
\hline Processing & 0.713 & 0.983 & 0.839 & 10 \\
\hline Content & 0.749 & 0.983 & 0.851 & 10 \\
\hline Performance & 0.821 & 0.984 & 0.815 & 10 \\
\hline Total & 0.778 & 0.986 & 0.873 & 10 \\
\hline
\end{tabular}

scenes, and each of the remaining 10 scenes shows a social interaction problem. The examiner stops playing the video after each scene and asks the examiner to answer a series of questions to assess the skills of receiving, processing, and sending.

\section{Reliability check}

Cronbach's alpha coefficient was used to determine the internal consistency of the test. To measure the reliability between the evaluator, two evaluators scored 22 patients during the test, and then, the correlation coefficient within the scores was calculated. To evaluate the relative reliability of the test, 15 patients were re-tested at intervals of 2 weeks, and the correlation coefficient within the range with a $95 \%$ confidence interval was used. Data were analyzed using SPSS software version 26.

\section{Results}

In this study, 52 patients with schizophrenia with a Mean \pm SD age and standard deviation of $39.92 \pm 10.32$, all of whom were male. Also, the Mean \pm SD length of hospital stay in these patients was 49.69 days with a standard deviation of 54.14 . Education $69.2 \%$ of the samples were below diploma and $30.8 \%$ were diploma and higher.

Validity

Regarding apparent validity, almost all the experts believed that the quality and clarity of the sound were not good in scenes 1 and 9; thus, the two scenes were filmed again. In other scenes, no particular problem was reported, they were approved and the test was performed without problems on 3 healthy people and 2 patients. According to 7 psychiatrists, the value of CVI and CVR for all scales was 1 .

\section{Reliability}

Cronbach's alpha coefficient was used to determine the internal consistency of the test. According to Table 1, Cronbach's alpha coefficient for different scales of the test was obtained between 0.511 and 0.821 .

As shown in Table 1, the intra-rank correlation coefficient for the two testers at different test scales ranged from 0.98 to 0.99 , indicating high reliability between the test evaluators. The correlation coefficient to calculate the repeatability of the test after two weeks in 15 samples for different test dimensions was between 0.733 and 0.893 (Table 1).

\section{Discussion and Conclusion}

The validity of the test content was obtained by providing the test to 7 experts and the CVI and CVR results were equal to one. Considering that the acceptable value for CVI is 0.79 and for CVR is 0.99 , the results of this study showed that this tool has an accepted content validity [31].

Cronbach's alpha coefficient was used to determine the internal consistency of the test. According to Table 1, Cronbach's alpha coefficient for different scales of the test was obtained between 0.511 and 0.821 , which indicates the appropriate internal consistency of the test. Donahue et al. obtained Cronbach's alpha coefficient of $0.65-0.78$ for different scales of the test [32], which is close to our results. Leung et al. (2006) also reported Cronbach's alpha of 0.766 for receiving and 0.982 for sending in a study on the Chinese version [23]. As mentioned, the response in each scale is influenced by the response of the previous scale, i.e. the individual's score in each scale depends to a large extent on his success rate in the previous scale. Therefore, the scales are not completely pure in the process of processing and 
sending. This can be a reason for the low Cronbach's alpha level and as a result, the internal consistency of the test.

\section{Results}

In this study, the reliability between the evaluators was evaluated by two testers on 22 patients. According to Table 1 , the correlation coefficient for all test domains was above 0.98. According to the classification, correlations less than $0.50,0.50-0.75$, and more than 0.75 are considered weak, medium, and good, respectively, and correlations above 0.9 are excellent [33]. As a result, the reliability between the evaluators in this study was considered excellent for all scales. In the study by Donahue et al. (1990), the reliability between the evaluators was assessed on 9 samples and the kappa coefficient for different test domains was calculated between 0.76 and 0.85 [32]. In a study using the Chinese version, Leung et al. (2006) used the Pearson correlation coefficient to evaluate the reliability of the evaluators and the percentage of agreement between the two testers for different test scales ranged from 0.871 to 0.986 [23]. In a study conducted on 20 French patients with schizophrenia, an agreement between two testers in different scales ranged from 0.90 to 0.95 [21]. In a study conducted on a Swedish version by Stalberg et al., the correlation between the two testers in different test scales was reported to be between 0.74 and 0.80 [24]. The results of the mentioned studies were consistent with the present study and showed the reliability between the appropriate evaluators of the test.

To evaluate the relative reliability of the test, the test was repeated on 15 patients at intervals of 2 weeks and the correlation coefficient for different domains of the test was between 0.733 and 0.893 (Table 1), which shows good reliability for all scales. In a study conducted by Donahue et al. (1990) on 9 samples with a time interval of 2 weeks from the first test, the second test was performed, the correlation range of the retest was reported from 0.46 to 0.77 [32]. In a study using the Chinese version, Leung et al. obtained the correlation of between 0.534 and 0.681 in the test-retest reliability for different domains [23]. One of the possible reasons for the higher reliability of the test in this study compared with the mentioned studies is that the test was performed on hospitalized patients in this study, while in other studies, and the test was performed on outpatients. Outpatients can perform better in testing due to symptom control; thus, they perform better in retesting. The results of this study and the studies mentioned above showed that the test has the appropriate reliability.

\section{Discussion and Conclusion}

Given the appropriate psychological conditions and characteristics of this tool mentioned earlier and the fact that the tool measures social skills based on the problem-solving model, this tool can be a good option for psychiatrists, especially occupational therapists. Because social participation is one of the factors of acupuncture in occupational therapy [34], this test should be used in the process of teaching social skills to schizophrenia patients.

Overall, the results obtained in this study showed that the Persian version of the tool for assessing interpersonal problem-solving skills has good validity and reliability and can be used for measuring social skills in Iranian patients with schizophrenia.

The most important limitation in this study was assessing hospitalized patients and the presence of acute symptoms in these patients possibly could affect the results. On the other hand, due to the existing limitations, this study was performed only on men and patients admitted to the studied hospital (Razi). The above limitations can affect the generalizability of this tool; thus, the results of this study should be generalized with more caution. Concerning these issues, it is suggested that further studies be conducted on women using a larger sample size to provide more accurate information.

\section{Ethical Considerations}

\section{Compliance with ethical guidelines}

This study was approved by the ethics committee of the University of Social Welfare and Rehabilitation Sciences (Code: OR.USER.REC.1397.081).

\section{Funding}

The present study was extracted from Ma. thesis of the first author at the Department of Occupational Therapy, University of Social Welfare and Rehabilitation Sciences.

\section{Authors' contributions}

Conceptualization: Milad Abolhassani, Ashraf Karbalaei Nouri; Validation: Milad Abolhassani, Mojtaba Khairkhah; Analysis, research and study: Milad Abolhassani, Mojtaba Khairkhah, Ashraf Karbalaei Nouri; Editing and finalizing: Milad Abolhassani; Supervision: Ashraf Karbalaei Nouri, Enayatullah Bakhshi; Data analysis and interpretation: Milad Abolhassani, Ashraf Karbalaei Nouri, Enayatullah Bakhshi. 


\section{Conflict of interest}

The authors declared no conflict of interest.

\section{Acknowledgments}

The authors would like to thank all the patients who participated in this study, the esteemed staff of the occupational therapy department of Razi Hospital, as well as all those who participated in the preparation of the Persian version of this tool. 


\title{
بررسى روايى و يايايي نسخه فارسى ابزار ارزيابى مهارتهاي حل مسئله بين فردى در اسكيزوفرنيا
}

\author{
ميلاد ابوالحسنى' (i)، "اشرف كربلايى نورى' (1)، عنايت الله بخشى' (1)، مجتبى خيرخواه
}

1. كروه كاردرماني، دائشكَاه علوم توائبخشى و سلامت اجتماعى، تهران، ايران.

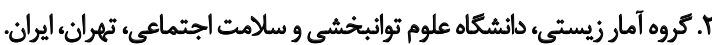

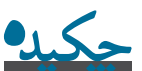

هدف هدف اين مطالعه ترجمه ابزار ارزيابى حل مسئلى بين فرديى و بروسى روائي و باياييى نسخهي فارسي اين آزمون بر روى بيماران سكيزوفرنى است.

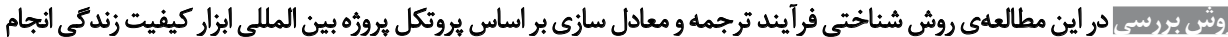

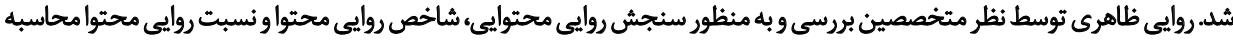

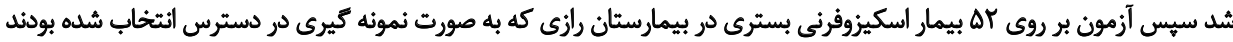

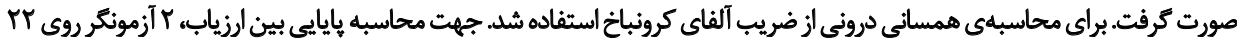

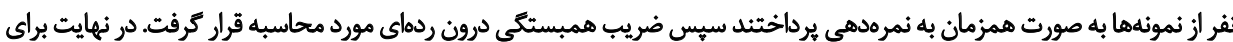

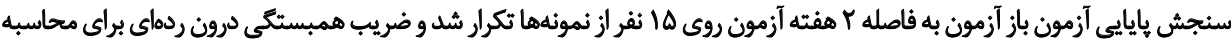

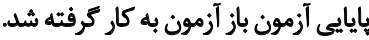

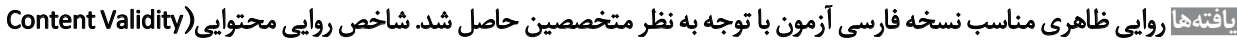

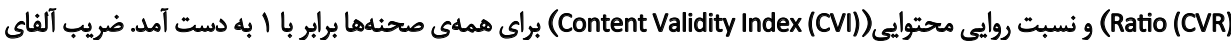

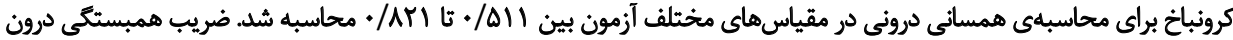

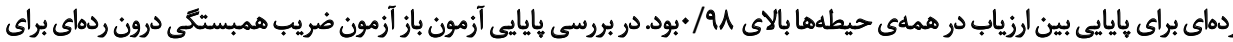

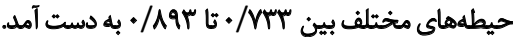

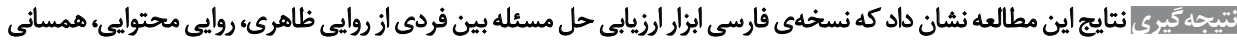

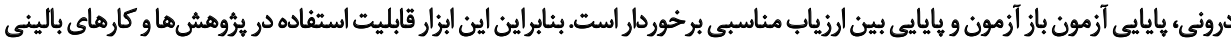

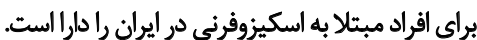

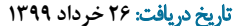

تاريخ يذيرش: م• آبان

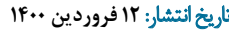

كليدوأرهاها:

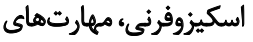

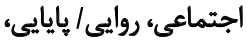

ترجمه

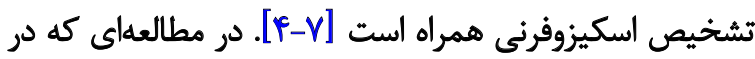

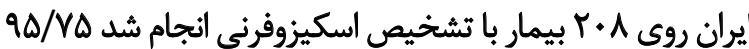

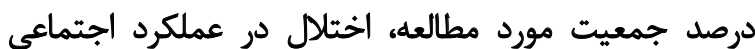

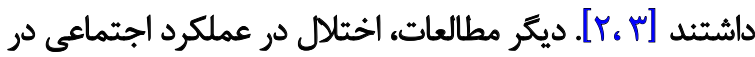

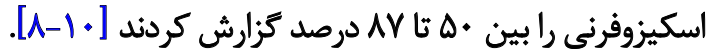
تملكرد اجتماعى يك وارثى كلى است كه به توانايى فرد براى

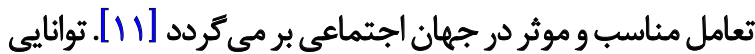

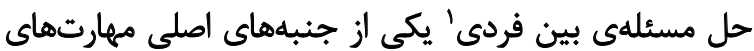

\section{Interpersonal Problem Solving}

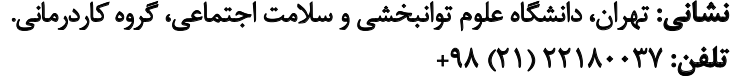

$$
\begin{aligned}
& \text { رايانامه: nouri.uswr@gmail.com }
\end{aligned}
$$

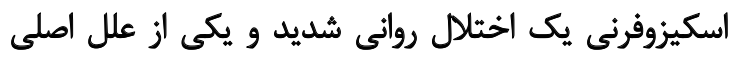

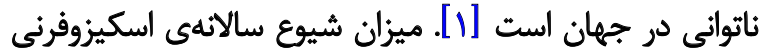

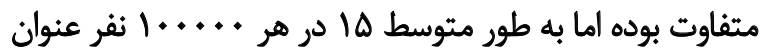

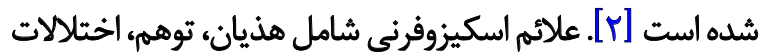

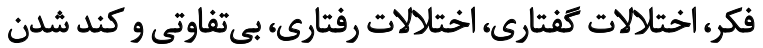

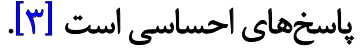

نقص در عملكرد اجتماعى و مهارتهاى اجتماعى اغلب با 


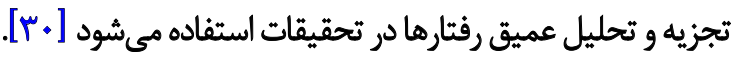

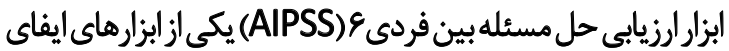

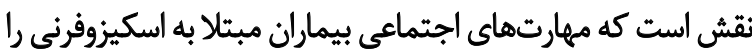

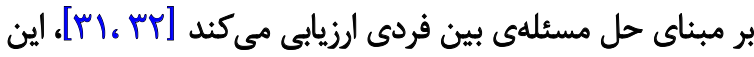

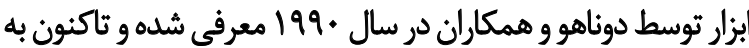

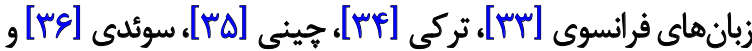

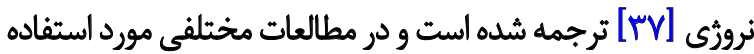

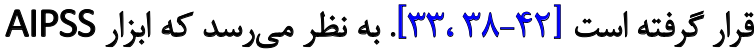

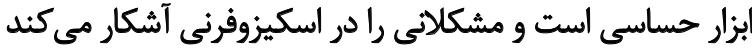

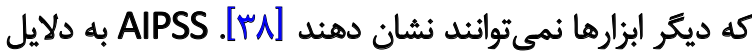

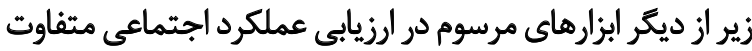

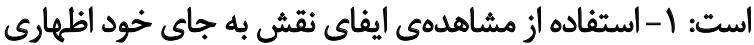

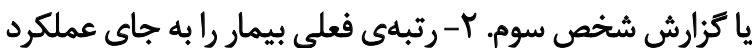

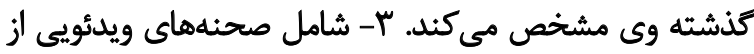

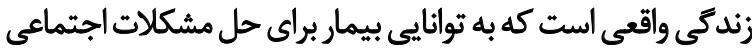

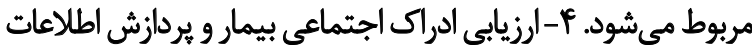

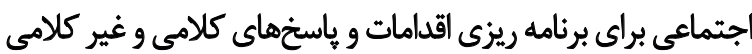

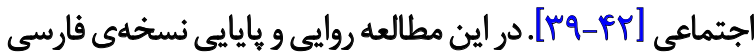
اين ابزار مورد بررسى قرار كرفته است.

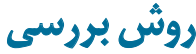

اين مطالعه از نوع روش شناختى (ابزار سازى، روان سنجى)

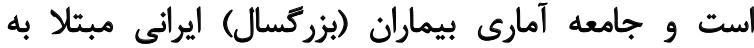

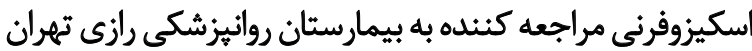

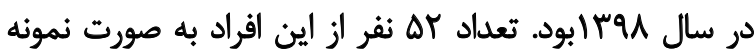

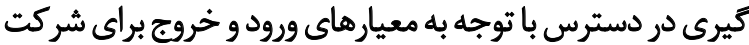

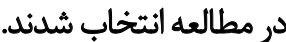

معيارهاى ورود شامل: 1 - ابتلا به اسكيزوفرنيا بر اساس نسخه

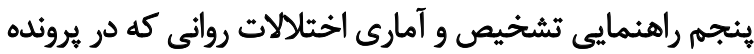

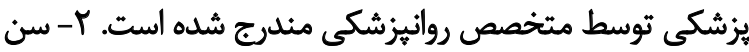

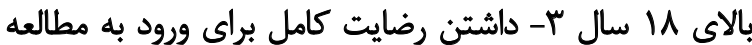

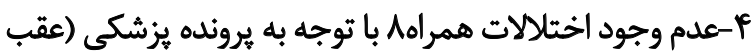

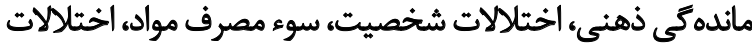

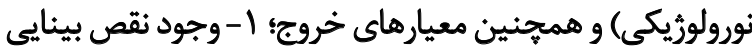

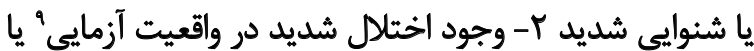

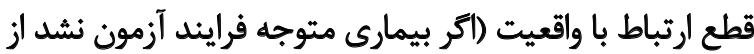

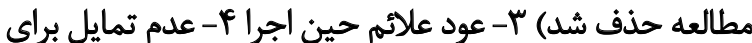

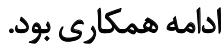

ياقْتهها

6. Assessment of Interpersonal Problem Solving (AIPSS)

7. Methodological

8. Comorbidity

9. Reality testing
اجتماعى است كه بسيارى از بيماران مبتلا به اسكيزوفرنى در آن

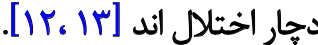

اختلال در عملكرد اجتماعى يكى از جنبههاى ناتوان كنيده

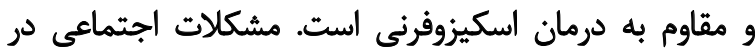

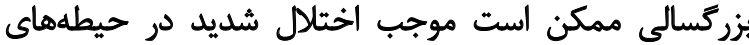

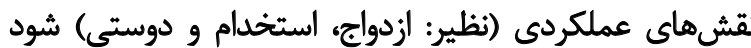

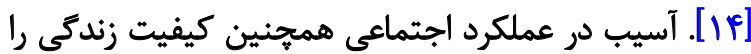

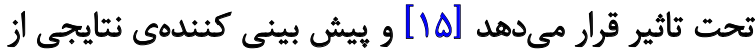

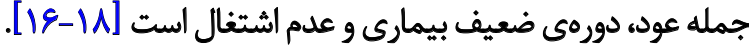

الروهاى ضد روانيريشى در حال حاضر اساس درمان

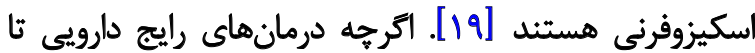

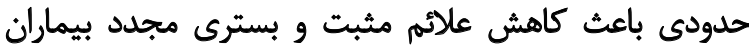

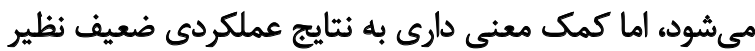

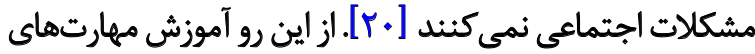

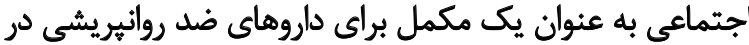

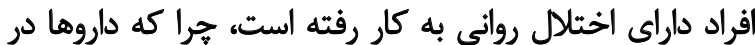

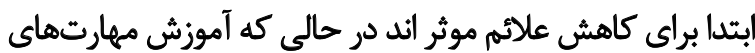

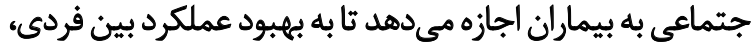

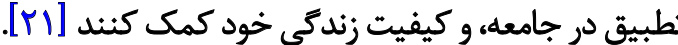

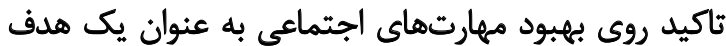

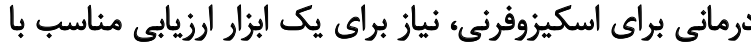

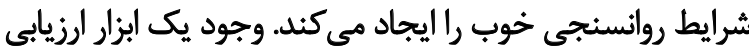

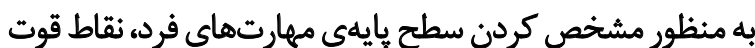

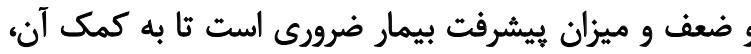
درمانكر برنامهى توانبخشى مناسبى را براي مراجع فراهم آورد.

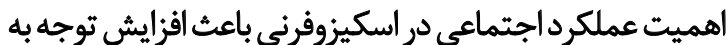

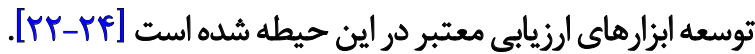

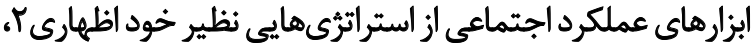

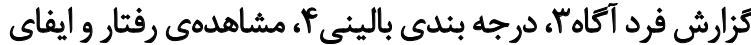

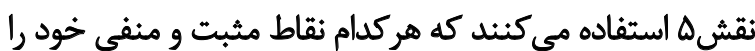

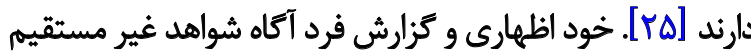

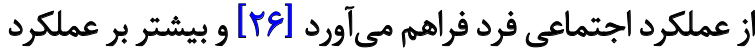

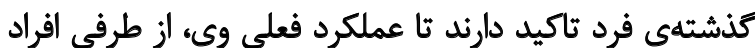

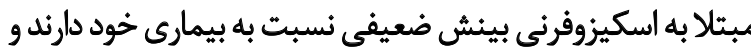

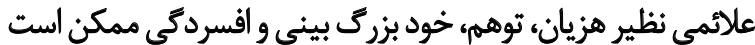

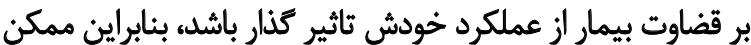

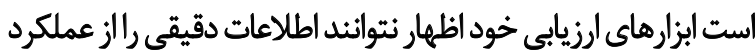

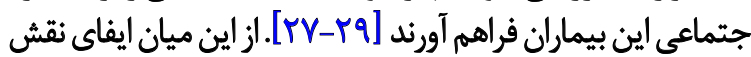

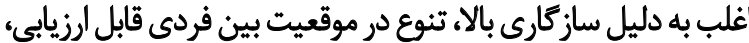

\section{Self-report \\ 3. Informant report \\ 4. Clinical rating}

5. Role-play 
بررسى روايى ظاهرى صحنههاى ارزيابى آزمون، با استفاده از

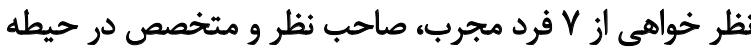

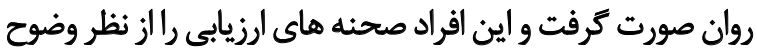

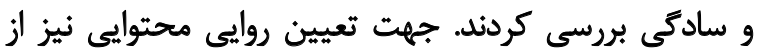

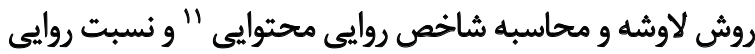

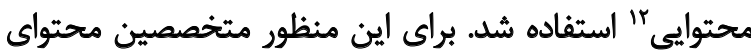

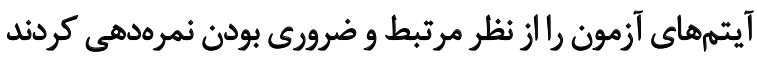

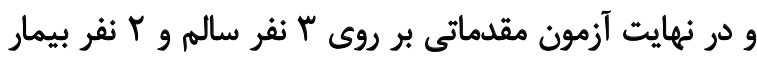

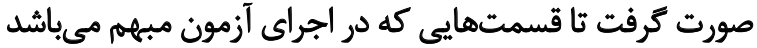

روشن شود.

إبزارها

در اين آزمون از يرسشنامه اطلاعات فردى و ابزار ارزيابى حل مسئلهى بين فردى استفاده شد.

\section{يرسشنامه اطلاعاث بين فردى}

اطلاعات دموكرافيك شامل سطح آموزش، جنس، سن،

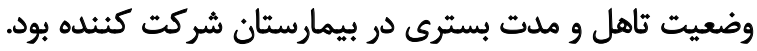

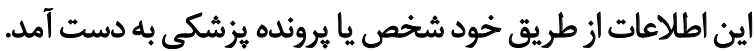

\section{إئار الوزيابى حل مسئله بين فردى}

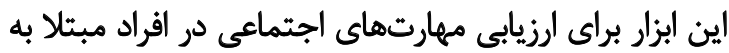

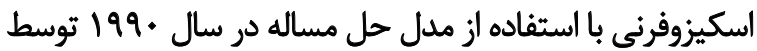

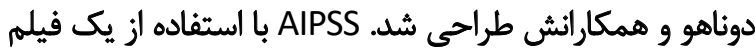

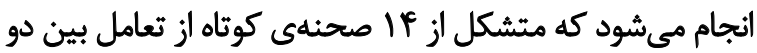

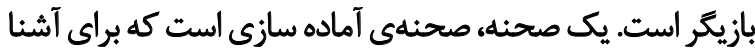

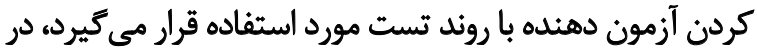

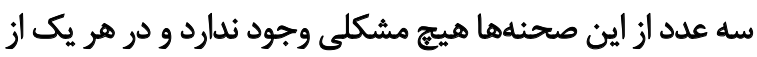

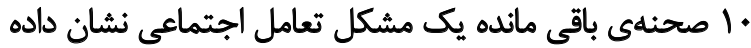

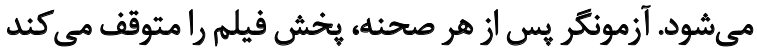

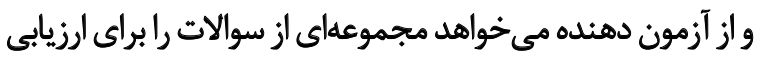
مهارتهاى دريافت، يردازش و ارسال ياسخ دهد.

AIPSS شش مقياس دارد: ابتداء دو مقياس براى مهارتهاى

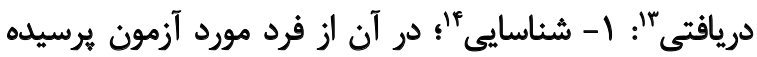

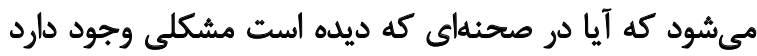

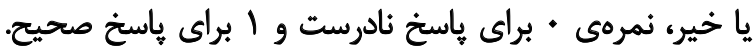

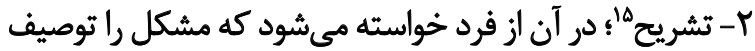

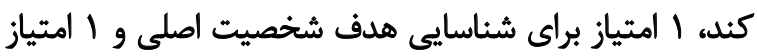
براى مشخص كردن مانع رسيدن به هدف. سيس يك مئ مقياس اصلي

11. Content Validity Ratio (CVR)

12. Content Validity Index (CVI)

13. Receiving skills

14. Identification

15. Description
اين مطالعه در دو مرحله كلى تهيهي نسخله فارسي و سيس

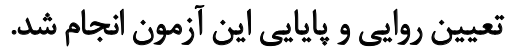

فرايند تهيه نسخه فارسى

زنسخي فارسي براساس بروتكلل بين المللى ارزيابي كيفيت

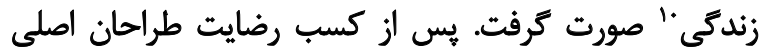

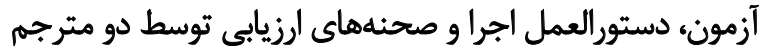

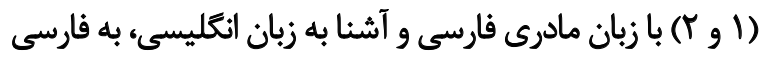

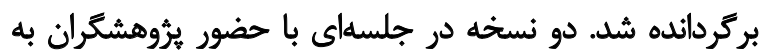

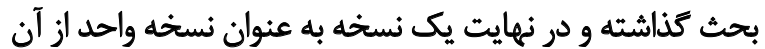

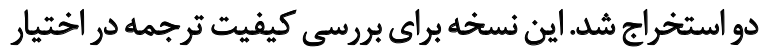

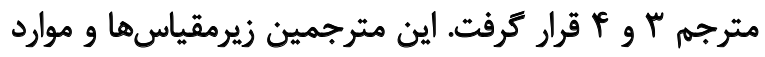

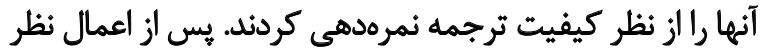

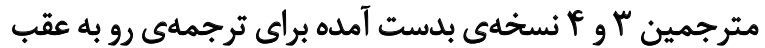

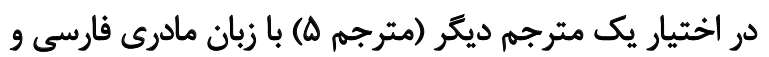

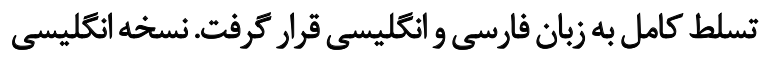

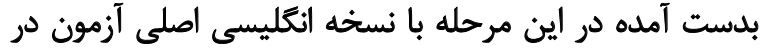

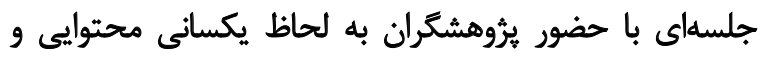

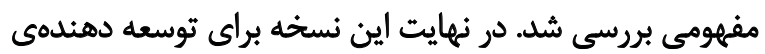

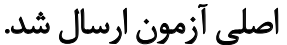

به منظور رسيدن به نسخهى فارسى صحنههاي ارزيابي ابتدا

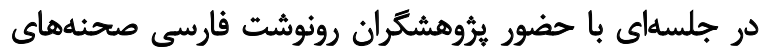

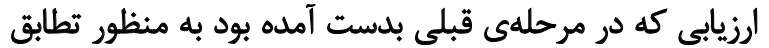

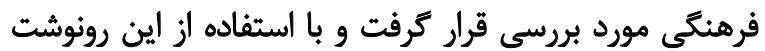

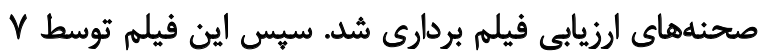

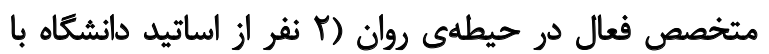

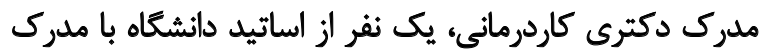

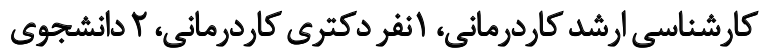

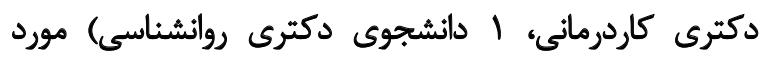

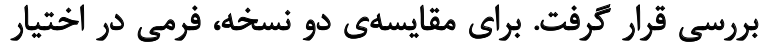

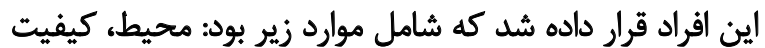

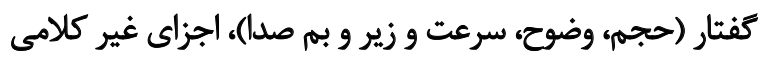

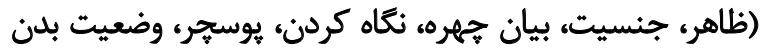

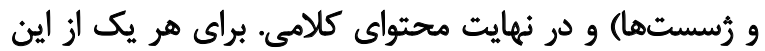

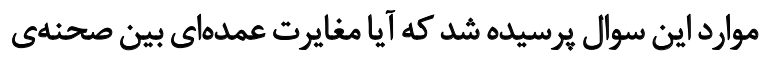

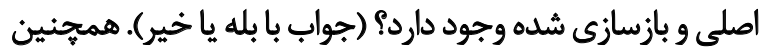

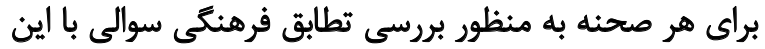

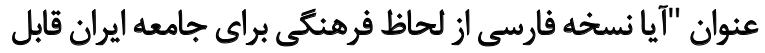

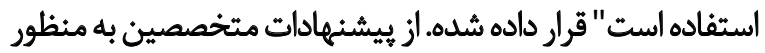
بهبود صحنههاى ارزيابي استفاده شد. بروسى روايي ظاهرى و محثوايي 10. The International Quality of Life Assessment 
كرفتيند. در ديكّر صحنهها مشكل خاصى عنوان نشد، مورد تاييد

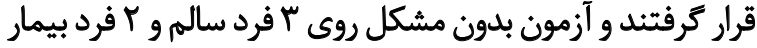

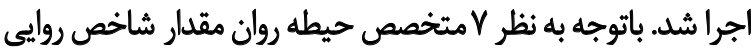

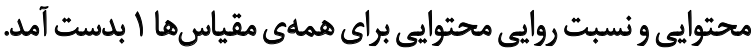

بايايهي

براي تعيين همسانى درونى آزمون از ضريب آلفاى كرونباخ

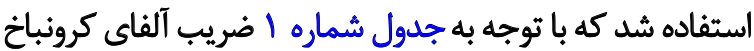

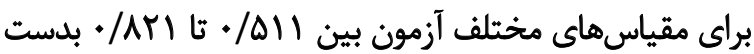

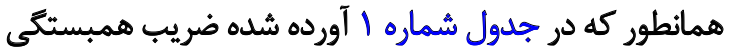

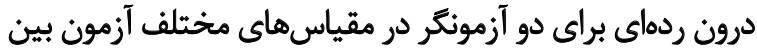

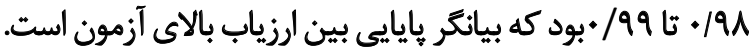

ضريب همبستكى درون ردماى حاصله در محاسبهى تكرار

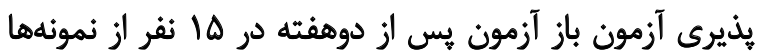

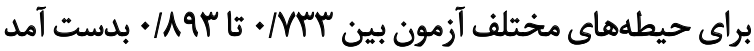

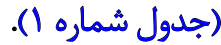

بحث

روايى محتواى آزمون با قرار دادن آزمون در اختيار V نفر از باز

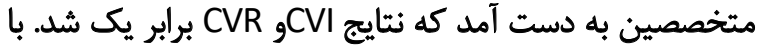

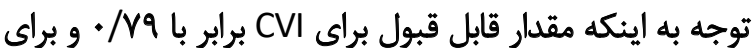

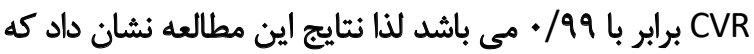

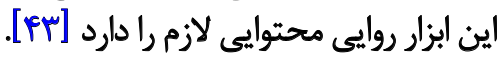

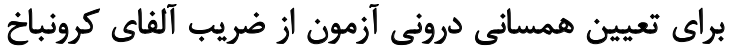

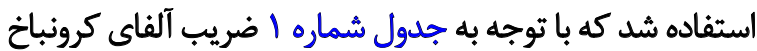

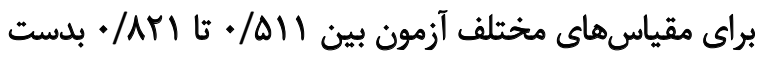

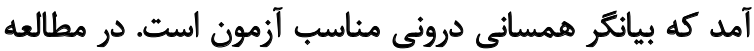

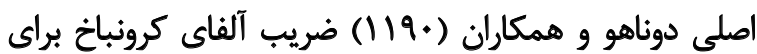

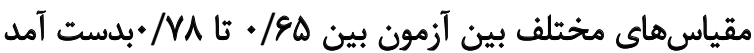

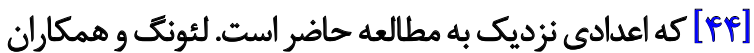

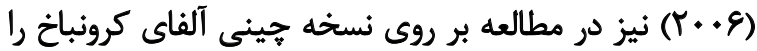

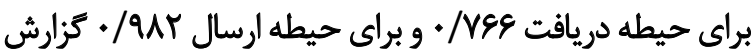

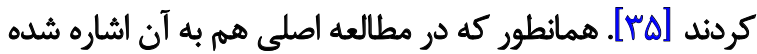

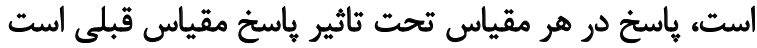

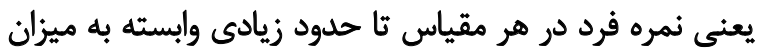

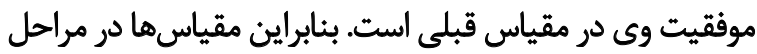

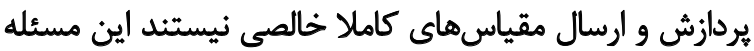

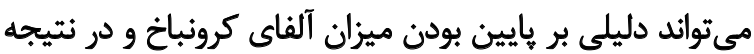
همسانى درونى آزمون باشد.

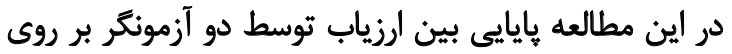

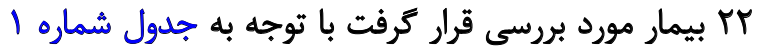

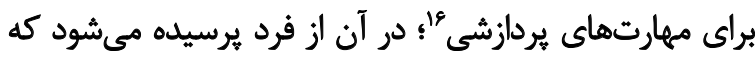

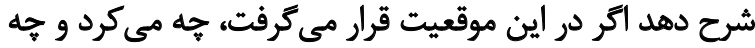

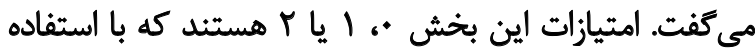

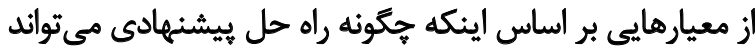

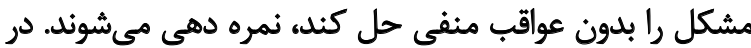

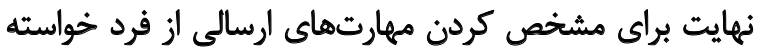

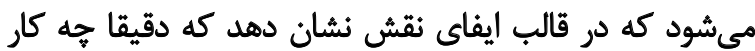

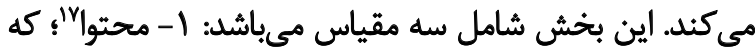

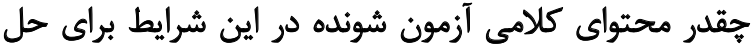

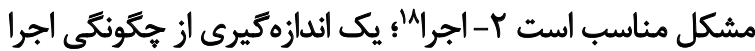

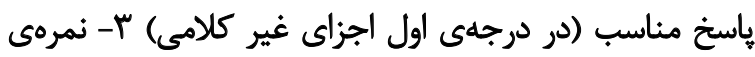

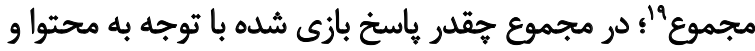

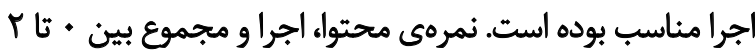

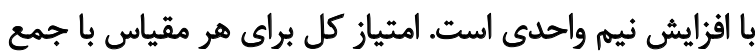

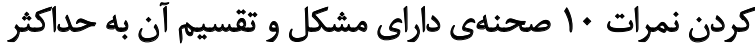

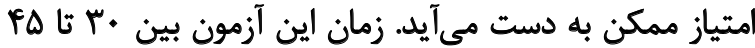

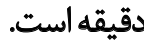

\section{بورسي بايانيي}

به منظور تعيين همسانى درونى آزمون از ضريب آلفاى بإي

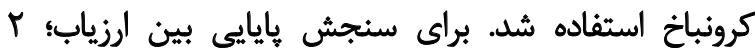

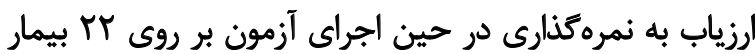

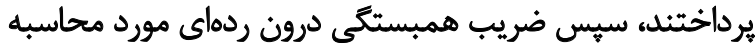

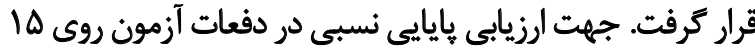

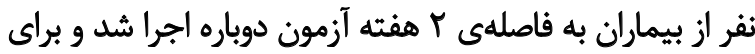

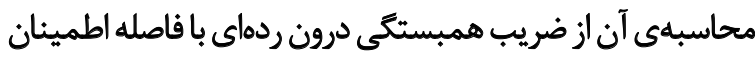

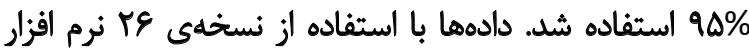
SPSS

ياقتهها

در اين مطالعه ه ه نفر از افراد مبتلا به اسكيزوفرنى با مياتكين

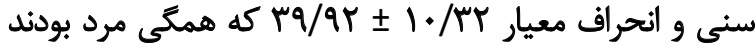

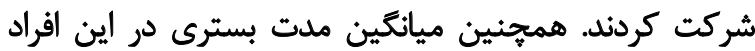

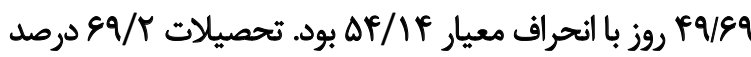

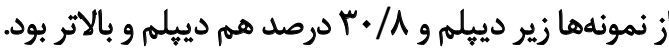

روايى

در بررسى روايى ظاهرى تقريبا بيشتر متخصصين بر اين نظر وفر ونا

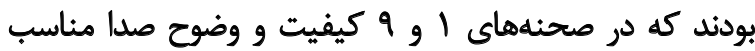
نيست، بر اين اساس اين دو صحنه دوباره مورد فيلم بردارى قرار

\section{Processing skills}

17. Content

18. Performance

19. Overall Sending Skills 
جدول ا. نتايج آلفاى كرونباخ و ضريب همبستكى درون ردهاى

\begin{tabular}{|c|c|c|c|c|}
\hline \multirow{2}{*}{ تعداد آيتمهها } & \multicolumn{2}{|c|}{ ضريب همبستكي درون درهاي } & \multirow{2}{*}{ آلفاى كرونباخ } & \multirow{2}{*}{ مقياس } \\
\hline & آزمون باز آزمون (تعداد= 10) & بين دو ارزياب (تعداد= Tr) & & \\
\hline ir & $\cdot M r$ & - /99 &.$/ 811$ & شناسايى \\
\hline 1. & ./194 & - /ur & ./EVA & تشريح \\
\hline 1. & ./Arq & . /ur & - Nir & يردازش \\
\hline 1. & - /ADI & ./ur & - Meq & محتوا \\
\hline 1. & $\cdot / A 18$ & - /ur & - AYI & اجرا \\
\hline 1. & $\cdot / A r r$ & $+/ 20$ & 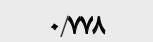 & مجموع \\
\hline
\end{tabular}

هايايى در دفعاث آزمون در اين مطالعه نسبث به مطالعاث ياد

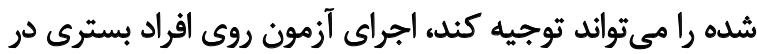

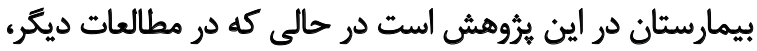

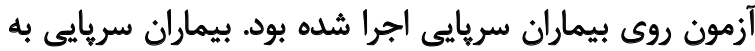

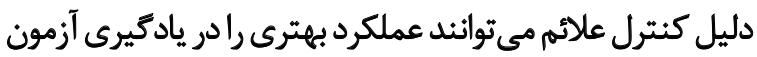

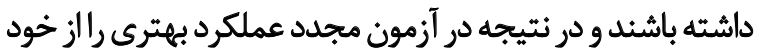
نشان دهند.نتايج اين مطالعه و مطالعات ذكر شده در در بالا نشان داد كه آزمون از هايايي آزمون باز آزمون مناسبي ماني برخودار است. باتوجه به شرايط روانسجى مناسب و ويزگى اين ابزار كه إنه

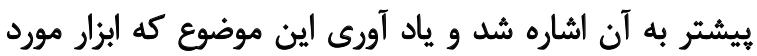

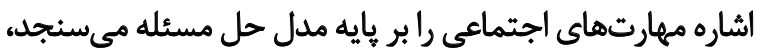

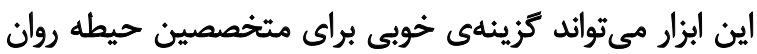

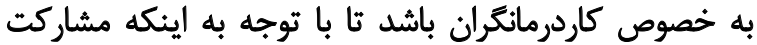

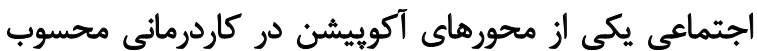

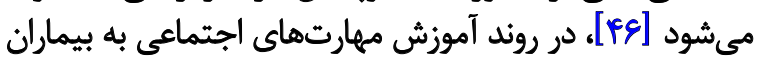
اسكيزوفرني مورد استفاده قرار كيرد. $5,5 d x$

در مجموع نتايج بدست آمده در اين مطالعه نشان داد كه

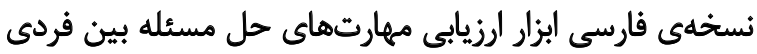

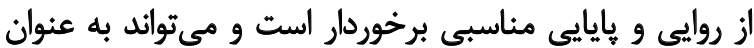
ابزار سنجش مهارتهاى اجتماعى در افراد فارسى زبان مبتلائ به اسكيزوفرنى در ايران به كار كرفته شود .

مهمترين محدوديت در اين مطالعه اين بود كه فقط بر روى دي بيماران بسترى انجام شد و وجود علائم حاد در اين بيماران

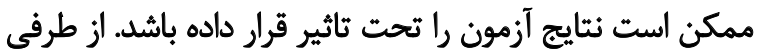
با توجه به محدوديتهاى موجود اين مطالعه فقط بر روى مردان

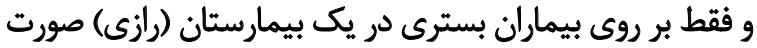

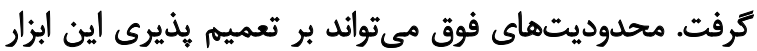

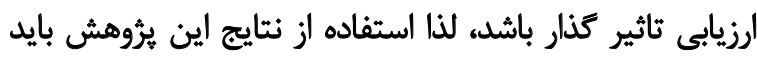

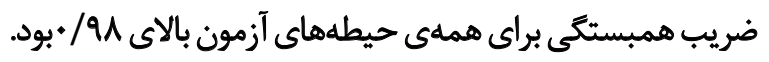

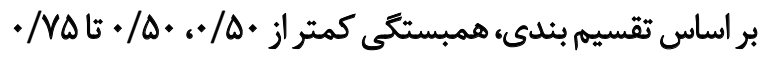

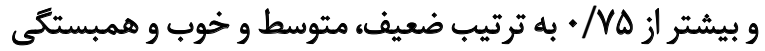

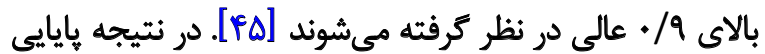

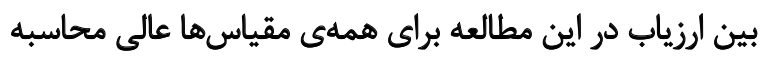

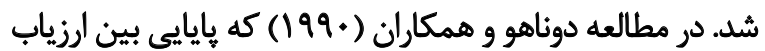

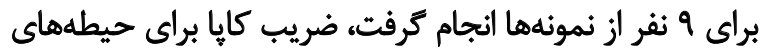

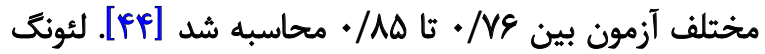

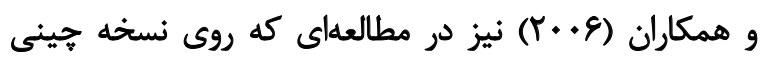

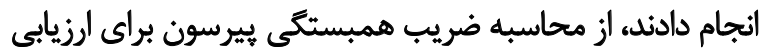

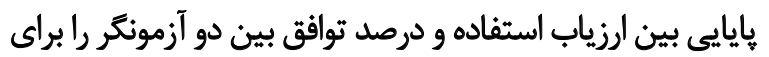

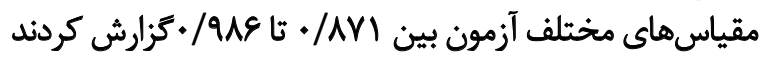

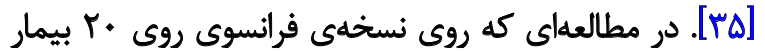

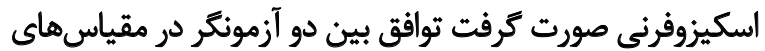

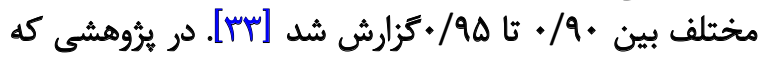
روى نسخهى سوئدى توسط استالبرى و همكاران صورت گترفت

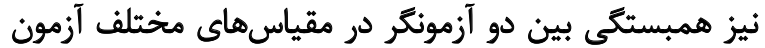

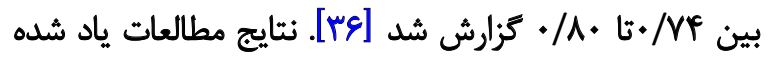

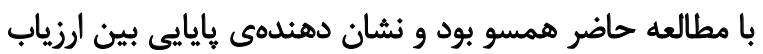
مناسب آزمون است. ماضر همبت جهت ارزيابى هايايى نسبى در دفعات آزمون روى 10 نفر

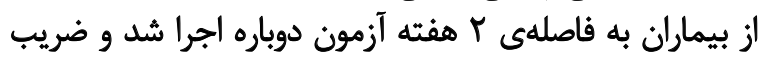

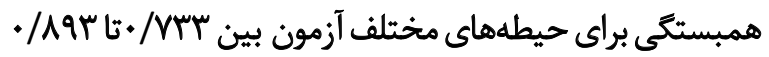

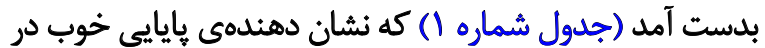

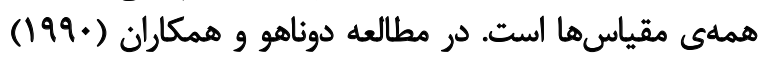

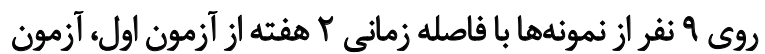

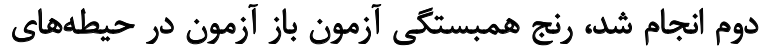

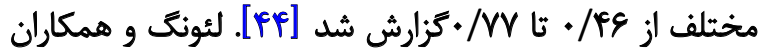

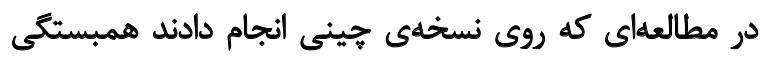

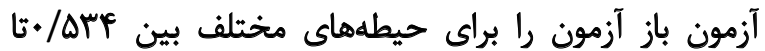

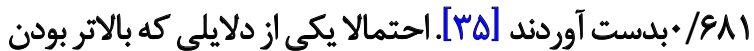


با احتياط بيشترى صورت كيرد. با توجه به اين مسائل بيشنهاد

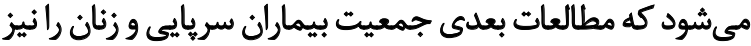

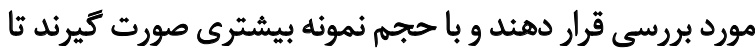
اطلاعات دقيقترى را فراهم سازند.

ماحظات اخالاقى

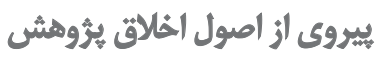

IR.USWR.REC.1397.081 كد اخلاق اين مطالعه به شناسه

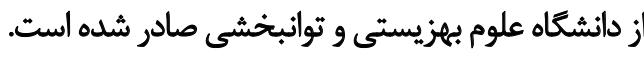

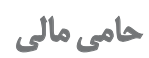

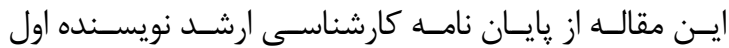

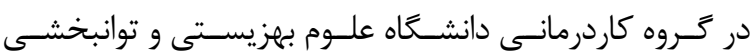

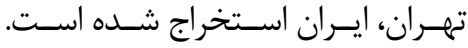

$$
\text { مشاركت نويسند مكان }
$$

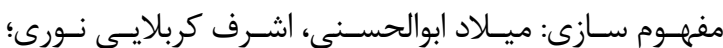

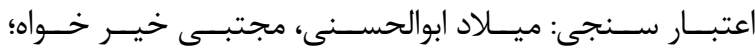

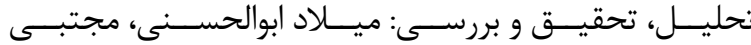

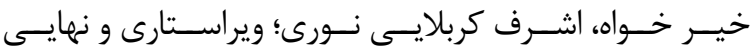

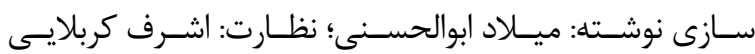

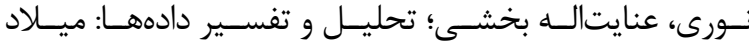

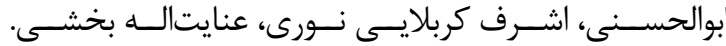

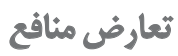

بنابر اظهار نويسندكان اين مقاله تعارض منافع ندارد.

$$
\text { تشكر وقدردانى }
$$

نويسندكان از تمام بيمارانى كه در اين مطالعه حضور داشتنده

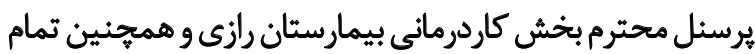

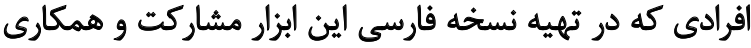
داشتند تشكر و قدردانى مى كنيند. 


\section{References}

[1] Hersen M, Turner SM, Beidel DC. Adult psychopathology and diagnosis. Hoboken, New Jersey: John Wiley \& Sons; 2011. https://books.google.com/books?id

[2] Tandon R, Keshavan MS, Nasrallah HA. Schizophrenia,"just the facts": what we know in 2008: part 1: Overview. Schizophrenia research. 2008; 100(1):4-19. [DOI:10.1016/j.schres.2008.01.022] [PMID]

[3] American Psychiatric Association. Diagnostic and Statistical Manual of mental disorders (DSM-5®). Philadelphia: American Psychiatric Pub; 2013. https://books.google.com/books?id

[4] Lysaker PH, Bell MD, Zito WS, Bioty SM. Social skills at work: Deficits and predictors of improvement in schizophrenia. The Journal of Nervous and Mental Disease. 1995; 183(11):688-92. [DOI:10.1097/00005053-199511000-00003] [PMID]

[5] Li F, Wang M. A behavioural training programme for chronic schizophrenic patients. The British Journal of Psychiatry. 1994; 165(S24):32-7. [DOI:10.1192/S0007125000292957]

[6] Haldford WK, Hayes RL. Social skills training with schizophrenic patients. In: Kavanagh D, editors. Schizophrenia: An overview and practical handbook. Londres: Chapman \& Hall; 1992. [DOI:10.1007/978-1-4899-4457-3_25]

[7] Gu Y, Peng H, Dai J, Gao H, Yang X, Sheng J, et al. Evaluation of paliperidone on social function in patients with chronic schizophrenia. General Psychiatry. 2018; 31(2). [DOI:10.1136/ gpsych-2018-000011] [PMID] [PMCID]

[8] Ganev K. Long-term trends of symptoms and disability in schizophrenia and related disorders. Social Psychiatry and Psychiatric Epidemiology. 2000; 35(9):389-95. [DOI:10.1007/ s001270050255] [PMID]

[9] Maziade M, Gingras N, Rodrigue C, Bouchard S, Cardinal A, Gauthier B, et al. Long-term stability of diagnosis and symptom dimensions in a systematic sample of patients with onset of schizophrenia in childhood and early adolescence. I: Nosology, sex and age of onset. The British Journal of Psychiatry. 1996; 169(3):361-70. [DOI:10.1192/bjp.169.3.361] [PMID]

[10] Ran M, Xiang M, Huang M, Shan Y. Natural course of schizophrenia: 2-year follow-up study in a rural Chinese community. The British Journal of Psychiatry. 2001; 178(2):154-8. [DOI:10.1192/ bjp.178.2.154] [PMID]

[11] Hooley JM. Social factors in schizophrenia. Current Directions in Psychological Science. 2010; 19(4):238-42. [DOI:10.1177/0963721410377597]

[12] Platt JJ, Spivack G. Social competence and effective problem-solving thinking in psychiatric patients. Journal of Clinical Psychology. 1972; 28(1):3-5. [DOI:10.1002/10974679(197201)28:13.0.CO;2-R]

[13] Hansen DJ, St Lawrence JS, Christoff KA. Effects of interpersonal problem-solving training with chronic aftercare patients on problem-solving component skills and effectiveness of solutions. Journal of Consulting and Clinical Psychology. 1985; 53(2):167. [DOI:10.1037/0022-006X.53.2.167] [PMID]
[14] Beels CC. Social support and schizophrenia. Schizophrenia Bulletin. 1981; 7(1):58-72. [DOI:10.1093/schbul/7.1.58] [PMID]

[15] Penn DL, Corrigan PW, Bentall RP, Racenstein J, Newman L. Social cognition in schizophrenia. Psychological Bulletin. 1997, 121(1):114. [DOI:10.1037/0033-2909.121.1.114] [PMID]

[16] Perlick D, Stastny P, Mattis S, Teresi J. Contribution of family, cognitive and clinical dimensions to long-term outcome in schizophrenia. Schizophrenia Research. 1992; 6(3):257-65 [DOI:10.1016/0920-9964(92)90009-T]

[17] Tien AY, Eaton WW. Psychopathologic precursors and sociodemographic risk factors for the schizophrenia syndrome. Archives of General Psychiatry. 1992; 49(1):37-46. [DOI:10.1001/ archpsyc.1992.01820010037005] [PMID]

[18] Ritsner MS, Arbitman M, Lisker A, Ponizovsky AM. Ten-year quality of life outcomes among patients with schizophrenia and schizoaffective disorder II. Predictive value of psychosocial factors. Quality of Life Research. 2012; 21(6):1075-84 [DOI:10.1007/s11136-011-0015-4] [PMID]

[19] Lieberman JA, Stroup TS, McEvoy JP, Swartz MS, Rosenheck RA, Perkins DO, et al. Effectiveness of antipsychotic drugs in patients with chronic schizophrenia. The New England Journal of Medicine. 2005; 2005(353):1209-23. [DOI:10.1056/NEJMoa051688] [PMID]

[20] Priebe S. Social outcomes in schizophrenia. The British Journal of Psychiatry. 2007; 191(50):s15-s20. [DOI:10.1192/bjp.191.50. s15] [PMID]

[21] Liberman RP. Dissemination and adoption of social skills training: Social validation of an evidence-based treatment for the mentally disabled. Journal of Mental Health. 2007; 16(5):595-623. [DOI:10.1080/09638230701494902]

[22] Lanser I, Browne J, Pinkham AE, Harvey PD, Jarskog LF, Penn DL. Evaluating social skill in individuals with schizophrenia with the Brief Impression Questionnaire (BIQ). Psychiatry Research 2018; 269:38-44. [DOI:10.1016/j.psychres.2018.08.047] [PMID] [PMCID]

[23] Harvey PD, Raykov T, Twamley EW, Vella L, Heaton RK, Patterson TL. Validating the measurement of real-world functional outcomes: Phase I results of the VALERO study. American Journal of Psychiatry. 2011; 168(11):1195-201. [DOI:10.1176/appi. ajp.2011.10121723] [PMID] [PMCID]

[24] Leifker FR, Patterson TL, Heaton RK, Harvey PD. Validating measures of real-world outcome: The results of the VALERO expert survey and RAND panel. Schizophrenia Bulletin. 2011; 37(2):334-43. [DOI:10.1093/schbul/sbp044] [PMID] [PMCID]

[25] Bellack AS, Green MF, Cook JA, Fenton W, Harvey PD, Heaton RK, et al. Assessment of community functioning in people with schizophrenia and other severe mental illnesses: A white paper based on an NIMH-sponsored workshop. Schizophrenia Bulletin. 2006; 33(3):805-22. [DOI:10.1093/schbul/sbl035] [PMID] [PMCID]

[26] Klonsky ED, Oltmanns TF. Informant-reports of personality disorder: Relation to self-reports and future research directions. Clinical Psychology: Science and Practice. 2002; 9(3):300-11. [DOI:10.1093/clipsy.9.3.300] 
[27] Aleman A, Agrawal N, Morgan KD, David AS. Insight in psychosis and neuropsychological function. The British Journal of Psychiatry. 2006; 189(3):204-12. [DOI:10.1192/bjp.189.3.204] [PMID]

[28] Harvey PD, Twamley EW, Pinkham AE, Depp CA, Patterson TL. Depression in schizophrenia: associations with cognition, functional capacity, everyday functioning, and self-assessment. Schizophrenia Bulletin. 2017; 43(3):575-82. [DOI:10.1093/schbul/sbw103] [PMID] [PMCID]

[29] Sabbag S, Twamley EW, Vella L, Heaton RK, Patterson TL, Harvey PD. Predictors of the accuracy of self assessment of everyday functioning in people with schizophrenia. Schizophrenia Research. 2012; 137(1-3):190-5. [DOI:10.1016/j.schres.2012.02.002] [PMID] [PMCID]

[30] Inderbitzen HM. Adolescent peer social competence. Advances in clinical child psychology. Springer; 1994. [DOI:10.1007/9781-4757-9041-2_8]

[31] Wallace CJ, Nelson CJ, Liberman RP, Aitchison RA, Lukoff D, Elder JP, et al. A review and critique of social skills training with schizophrenic patients. Schizophrenia Bulletin. 1980; 6(1):42-63. [DOI:10.1093/schbul/6.1.42] [PMID]

[32] Liberman RP, Mueser KT, Wallace CJ, Jacobs HE, Eckman T, Massel HK. Training skills in the psychiatrically disabled: learning coping and competence. Schizophrenia Bulletin. 1986; 12(4):631-47. [DOI:10.1093/schbul/12.4.631] [PMID]

[33] Zanello A, Perrig L, Huguelet P. Cognitive functions related to interpersonal problem-solving skills in schizophrenic patients compared with healthy subjects. Psychiatry Research. 2006; 142(1):67-78. [DOI:10.1016/i.psychres.2003.07.009] [PMID]

[34] Üçok, A., Öztürk, M., Duman, Z., \& Saruhan-Direskeneli, G. (2010). COMT Val158Met polymorphism is related with interpersonal problem solving in schizophrenia. European Psychiatry, 25(6):320-22. [DOI:10.1016/j.eurpsy.2010.01.012]

[35] Leung TK, Tsang HW. Chinese version of the assessment of interpersonal problem solving skills. Psychiatry Research. 2006; 143(2):189-97. [DOI:10.1016/j.psychres.2005.08.011] [PMID]

[36] Stålberg G, Lichtenstein P, Sandin S, Hultman CM. Videobased assessment of interpersonal problem solving skills in patients with schizophrenia, their siblings and non-psychiatric controls. Scandinavian Journal of Psychology. 2008; 49(1):77-82. [DOI:10.1111/j.1467-9450.2007.00615.x] [PMID]

[37] Vaskinn A, Sundet K, Friis S, Simonsen C, Birkenaes AB, Jonsdottir $\mathrm{H}$, et al. Emotion perception and learning potential: Mediators between neurocognition and social problem-solving in schizophrenia? Journal of the International Neuropsychological Society. 2008; 14(2):279-88. [DOI:10.1017/S1355617708080314] [PMID]

[38] Vaskinn A, Sundet K, Hultman CM, Friis S, Andreassen OA. Social problem-solving in high-functioning schizophrenia: Specific deficits in sending skills. Psychiatry Research. 2009; 165(3):215-23. [DOI:10.1016/j.psychres.2007.11.009] [PMID]

[39] Sullivan G, Marder SR, Liberman RP, Donahoe CP, Mintz J. Social skills and relapse history in outpatient schizophrenics. Psychiatry. 1990; 53(4):340-5. [DOI:10.1080/00332747.1990.11024518] [PMID]
[40] Struchen MA, Pappadis MR, Sander AM, Burrows CS, Myszka KA. Examining the contribution of social communication abilities and affective/behavioral functioning to social integration outcomes for adults with traumatic brain injury. The Journal of Head Trauma Rehabilitation. 2011; 26(1):30-42. [DOI:10.1097/ HTR.0b013e3182048f7c] [PMID]

[41] Corrigan PW, Toomey R. Interpersonal problem solving and information processing in schizophrenia. Schizophrenia Bulletin. 1995; 21(3):395. [DOI:10.1093/schbul/21.3.395] [PMID]

[42] Combs DR, Finn JA, Wohlfahrt W, Penn DL, Basso MR. Social cognition and social functioning in nonclinical paranoia. Cognitive Neuropsychiatry. 2013; 18(6):531-48. [DOI:10.1080/135468 05.2013.766595] [PMID]

[43] Taghizadeh Z, Ebadi A, Montazeri A, Shahvari Z, Tavousi M, Bagherzadeh R. [Psychometric properties of health related measures. Part 1: Translation, development, and content and face validity (Persian)]. Payesh (Health Monitor). 2017; 16(3):343-57. http://payeshjournal.ir/article-1-108-en.html

[44] Donahoe CP, Carter MJ, Bloem WD, Hirsch GL, Laasi N, Wallace CJ. Assessment of interpersonal problem-solving skills. Psychiatry. 1990; 53(4):329-39. [DOI:10.1080/00332747.1990.11024 517] [PMID]

[45] Koo TK, Li MY. A guideline of selecting and reporting intraclass correlation coefficients for reliability research. Journal of Chiropractic Medicine. 2016; 15(2):155-63. [DOI:10.1016/j. jcm.2016.02.012] [PMID] [PMCID]

[46] Amini DA, Kannenberg K, Bodison S, Chang P, Colaianni D, Goodrich B, et al. Occupational therapy practice framework: Domain \& process $3^{\text {rd }}$ edition. American Journal of Occupational Therapy. 2014; 68:S1-S48. [DOI:10.5014/ajot.2014.682006] 
This Page Intentionally Left Blank 